\title{
IMPLEMENTATION OF THE SMOOTHED PARTICLE HYDRODYNAMICS METHOD TO SOLVE PLASTIC DEFORMATION IN METALS
}

\author{
${ }^{1,2}$ E.A. Patiño, ${ }^{2}$ R. Reyes, ${ }^{2}$ D.A. García, ${ }^{2}$ A.F. Sarmiento, ${ }^{2}$ J.M. Arroyo, D.A. Garzon ${ }^{2}$. \\ ${ }^{1}$ Corresponding author (eapatinon@unal.edu.co) \\ ${ }^{2}$ Department of Mechanical and Mechatronics Engineering, National University of Colombia, \\ Bogotá.
}

\begin{abstract}
In this work it was implemented the numerical method of Smoothed Particle Hydrodynamics (SPH) to solve plastic deformation problems. The SPH is a meshfree particle method, based on a Lagrangian formulation; this is a computationally efficient method that provides precision and stable solutions to integral and differential equations. This formulation includes the continuum mechanics equations for solids, modified by the Johnson-Cook model to represent the plastic behavior of metallic materials. Benchmarks problems and experimental validation are provided.
\end{abstract}

Keywords: Smoothed Particle Hydrodynamics; Plastic Deformation.

\section{INTRODUCTION}

Smoothed Particle Hydrodynamics (SPH) is a technique developed for solving Computational Continuum Dynamics problems. Thus, SPH is a mesh free method based on a Lagrangian approximation for solving partial differential equation systems.

It was used first in Astrophysics problems [1], but nowadays SPH is applied in many fluid and solid mechanics problems [2-4]. The basic SPH technique was first introduced by Lucy, Gingold and Monaghan in 1997 [5], however the most important works in materials strength were provided by Libersky and Petshek [6]. Since then, many authors have implemented SPH in solid mechanics, and lot of papers present applications on numerical fracture [7], elastic [8] and plastic [9] regime deformations in solids, extreme deformation and material processing applications (extrusion, forging and machining) [10], [11].

The application of SPH method in solid mechanics uses the conservation equations of continuum mechanics with energy, momentum and density equations. This work includes lineal terms (elastic deformation) using Hooke's law for the stress tensor, and to describe the non-diagonal terms of the stress tensor this is modified by a von Mises yielding relation, when beyond the elastic limit for permanent deformation. Plasticity is treated using the Johnson and Cook visco-plastic model [12] and an elastic - plastic perfect model was used for the stress and deformation behavior.

This paper presents the application of SPH method to simulate large strains with material strength. A SPH code was implemented and evaluated in three test problems, the impact of a plate against a rigid surface, high shear in a plate and the penetration of a cylinder through a plate. The three example problems are compared using other SPH modeling of the 
literature and the experimental data. The results that were obtained in the different simulations presents an adequate behavior compared to the experimental and other simulation sources.

\section{SPH METHODS}

A little summary of the SPH method is presented here. For more details see [13]. The basis of SPH is interpolation theory, and its formulation involves two steps. In the first step the representation of a function in its continuous form is done through the use of a kernel estimate of the field variables at a point [14], this approximation of the function is based on the evaluation of the smoothing kernel function. Then, the second step is the representation of the interpolation kernel over the defined domain using discrete variables [15].

Then, the interpolated value of a function $f$ in the space defined by the $\mathbf{x}$ vector can be expressed using SPH smoothing as (1).

$$
\left\langle f\left(\mathbf{x}_{i}\right)\right\rangle=\sum_{j=1}^{N} \frac{m_{j}}{\rho_{j}} f_{j} W\left(\mathbf{x}_{i}-\mathbf{x}_{j}, h\right)
$$

Where $m_{j}$ and $\rho_{j}$ are the mass and density of particle $j, W\left(\mathbf{x}_{i}-\mathbf{x}_{j}, h\right)$ is the smoothing kernel, $h$ is the smoothing length (see more in [4]) and $N$ is the total number of particle. The gradient of function $f$ can also be found by differentiating the interpolation (1) as expressed in (2).

$$
\left\langle\nabla f\left(\mathbf{x}_{i}\right)\right\rangle=\sum_{j=1}^{N} \frac{m_{j}}{\rho_{j}} f_{j} \nabla_{i} W\left(\mathbf{x}_{i}-\mathbf{x}_{j}, h\right)
$$

Definition of the kernel function is represented in (3).

$$
W\left(\mathbf{x}_{i}-\mathbf{x}_{j}, h\right)=\frac{1}{h^{d}} \varphi\left(\frac{\left\|\mathbf{x}_{i}-\mathbf{x}_{j}\right\|}{h}\right)=W_{i j}
$$

Where $\varphi$ is the function that describes kernel approximation and $d$ is the number of space dimensions. Although there are several smoothing functions widely used, it is usually preferred the cubic B-spline [16], [17], which is defined by (4).

$$
W(q, h)=\alpha_{d} \begin{cases}\frac{2}{3}-q^{2}+\frac{1}{2} q^{3} & 0 \leq q<1 \\ \frac{1}{6}(2-q)^{3} & 1 \leq q<2 \\ 0 & 2 \leq q\end{cases}
$$


Where $\alpha_{d}$ is a constant of normalization which depends on the number of spatial dimensions (for one dimension is $\alpha_{1 D}=\frac{1}{h}$, for two dimensions is $\alpha_{2 D}=\frac{15}{7 \pi h^{2}}$ and for three dimensions is $\alpha_{3 D}=\frac{3}{2 \pi h^{3}}$ ), where $\left\|\mathbf{x}_{i}-\mathbf{x}_{j}\right\|$ is the distance between two particles.

\section{IMPLEMENTATION OF THE ELASTIC-PERFECTLY PLASTIC STRENGTH MODEL}

The SPH method applied in solids mechanics, integrates the traditional equations of mass (5), momentum (6) and energy (7).

$$
\begin{aligned}
& \frac{d \rho}{d t}=-\rho(\nabla \cdot \mathbf{v}) \\
& \frac{d \mathbf{v}}{d t}=\frac{1}{\rho} \nabla \cdot \boldsymbol{\sigma}+\mathbf{F} \\
& \frac{d E}{d t}=\frac{1}{\rho} \boldsymbol{\sigma}: \nabla^{s} \mathbf{v}=\frac{1}{\rho} \boldsymbol{\sigma}:\left[\frac{1}{2}(\nabla \otimes \mathbf{v}+\mathbf{v} \otimes \nabla)\right]+Q_{i j}
\end{aligned}
$$

Where for the equations (5) to (7), $\rho$ is density, $\mathbf{v}$ is the velocity vector ( $v$ is also defined as $d x / d t), \boldsymbol{\sigma}$ is the stress tensor, $\mathbf{F}$ is external force, $E$ is energy, $Q_{i j}$ is the heat flux, $\nabla$ is the gradient, $\nabla^{s}$ is a symmetric gradient, $T$ is the temperature and $t$ is the time. With the above definitions, the conservation equations can be written in their discrete form, then the equation of conservation of mass, momentum and energy can be transformed into particle equations for SPH with (8), (9) and (10) respectively [15], [18].

$$
\begin{aligned}
\left\langle\frac{d \rho}{d t}\right\rangle & =\rho_{i} \sum_{j=1}^{N} \frac{m_{j}}{\rho_{j}}\left(\mathbf{v}_{j}-\mathbf{v}_{i}\right) \cdot \nabla_{i} W_{i j} \\
\left\langle\frac{d \mathbf{v}}{d t}\right\rangle & =\sum_{j=1}^{N} m_{j}\left(\frac{\boldsymbol{\sigma}_{j}}{\rho_{j}^{2}}+\frac{\boldsymbol{\sigma}_{i}}{\rho_{i}^{2}}-\prod_{i j}\right) \cdot \nabla_{i} W_{i j}+\mathbf{F} \\
\left\langle\frac{d E}{d t}\right\rangle & =\frac{1}{2} \sum_{j=1}^{N} m_{j}\left(\frac{\boldsymbol{\sigma}_{i}}{\rho_{i}^{2}}+\frac{\boldsymbol{\sigma}_{j}}{\rho_{j}^{2}}-\prod_{i j}\right):\left[\left(\mathbf{v}_{j}-\mathbf{v}_{i}\right) \otimes \nabla_{i} W_{i j}+\nabla_{i} W_{i j} \otimes\left(\mathbf{v}_{j}-\mathbf{v}_{i}\right)\right]+Q_{i j}
\end{aligned}
$$

Where the brackets \langle\rangle are used to identify the function approximated by the kernel estimation, and $\prod_{i j}$ term represents the artificial viscous pressure.

The term of the heat flux is defined like (11), This form is used for [15], [19]. 


$$
\begin{aligned}
& Q_{i j}=\sum_{j=1}^{N} 4 \frac{m_{j}}{\rho_{i} \rho_{j}}\left(\frac{k_{i} k_{j}}{k_{i}+k_{j}}\right)\left(\frac{T_{i}-T_{j}}{\mathbf{r}_{i j} \cdot \mathbf{r}_{i j}}\right)\left(\mathbf{r}_{i j} \cdot \nabla_{i} W_{i j}\right) \\
& \mathbf{r}_{i j}=x_{i}^{\alpha}-x_{j}^{\alpha}
\end{aligned}
$$

Where in (11) and (12), $k$ is material conduction, $\mathbf{r}_{i j}$ is the distance vector between two particles and $x^{\alpha}$ is the component coordinate of vector $\mathbf{x}_{i}$.

The artificial viscous pressure was used as the Monaghan-Gingold form [17](13).

$$
\prod_{i j}= \begin{cases}\frac{-\alpha \bar{c}_{i j} \mu_{i j}^{2}+\beta \mu_{i j}^{2}}{\bar{\rho}_{i j}} & \text { if }\left(\mathbf{v}_{j}-\mathbf{v}_{i}\right) \cdot\left(\mathbf{x}_{j}-\mathbf{x}_{i}\right)<0 \\ 0 & \text { otherwise }\end{cases}
$$

Where $\mu_{i j}=\frac{\bar{h}_{i j}\left(\mathbf{v}_{j}-\mathbf{v}_{i}\right) \cdot\left(\mathbf{x}_{j}-\mathbf{x}_{i}\right)}{\left|\mathbf{x}_{j}-\mathbf{x}_{i}\right|^{2}+0,01 \bar{h}_{i j}^{2}}, \bar{c}_{i j}=\frac{\left(c_{i}+c_{j}\right)}{2}, \bar{\rho}_{i j}=\frac{\left(\rho_{i}+\rho_{j}\right)}{2}, \bar{h}_{i j}=\frac{\left(h_{i}+h_{j}\right)}{2}$ and the parameters $\alpha$ and $\beta$ are order unity and $c_{i}$ is the speed of sound on the ith particle.

\subsection{Equations for an elastic-perfectly plastic strength model}

The stress tensor appearing in the conservation equations (9) and (10) is defined in term of the pressure $P=-\frac{1}{3} \operatorname{Tr}(\boldsymbol{\sigma}) \mathbf{1}$ and the traceless symmetric deviatoric stress $\tilde{\sigma}$ as (14).

$$
\boldsymbol{\sigma}=\tilde{\boldsymbol{\sigma}}-P \mathbf{1}
$$

The pressure is normally computed using an state equation of the form $p=p(\rho, E)$, such as the Mie-Gruneisen equation for solids defined in (15).

$$
\begin{aligned}
& P(\rho, E)=\left(1-\frac{1}{2} \Gamma \eta\right)\left[P_{H}(\rho)\right]+\Gamma \rho E \\
& \eta=\frac{\rho}{\rho_{0}}-1 \\
& P_{H}(\rho)=\left\{\begin{array}{cc}
a_{0} \eta+b_{0} \eta^{2}+c_{0} \eta^{3} & , \eta>0 \\
a_{0} \eta & , \eta<0
\end{array}\right.
\end{aligned}
$$

Where for (15) the subscript $H$ refers to the Hugoniot curve and $\Gamma$ is the Gruneisen parameter and the constants $a_{0}, b_{0}, c_{0}$ can be definition with lineal relation between to shock velocity $U_{s}$ and particle velocity $S_{s}$, defined for $U_{s}=c+S_{s} U_{p}$. Through a Taylor's series expansion of the Hugoniot function the equations (16) are obtained. 


$$
\begin{aligned}
& a_{0}=\rho_{0} c_{s}^{2} \\
& b_{0}=a_{0}\left[1+2\left(S_{s}-1\right)\right] \\
& c_{0}=a_{0}\left[2\left(S_{s}-1\right)+3\left(S_{s}-1\right)^{2}\right]
\end{aligned}
$$

For the deviatoric part of equation (14), in the elastic range, we used Hooke's law as shown in (17).

$$
\begin{aligned}
& \dot{\tilde{\boldsymbol{\sigma}}}=2 \mu \dot{\tilde{\boldsymbol{\varepsilon}}} \\
& \dot{\tilde{\boldsymbol{\varepsilon}}}=\dot{\boldsymbol{\varepsilon}}-\frac{1}{3} \operatorname{tr}(\dot{\boldsymbol{\varepsilon}}) \mathbf{1} \\
& \dot{\boldsymbol{\varepsilon}}=\nabla^{S} \mathbf{v}=\frac{1}{2}(\nabla \otimes \mathbf{v}+\mathbf{v} \otimes \nabla)
\end{aligned}
$$

Where for equations (17) to (19), $\tilde{\boldsymbol{\sigma}}$ is the deviatoric stress rate tensor and $\dot{\boldsymbol{\varepsilon}}$ is the strain rate tensor and $\dot{\tilde{\boldsymbol{\varepsilon}}}$ is the strain rate deviatoric tensor. However, for finite rotations, the equations (17) is not material frame indifferent, then the rotation becomes dependent of a nonphysical response. To improve this situation many cases have been formulated [3]. In this work is employed the widely used Jaumann rate [2], [8] for small rotations and it alters (17) to appear as (20).

$$
\frac{d \tilde{\boldsymbol{\sigma}}}{d t}=2 \mu \dot{\tilde{\boldsymbol{\varepsilon}}}-\dot{\boldsymbol{\omega}} \cdot \tilde{\boldsymbol{\sigma}}-\tilde{\boldsymbol{\sigma}} \cdot \dot{\boldsymbol{\omega}}
$$

Where for the equation (20), $\dot{\boldsymbol{\omega}}$ is the rotational or spin rate strain tensor taken for (21), which it's defined by $\nabla^{A}$ that is the anti-symmetric gradient, and $\mu$ is the material shear modulus.

$$
\dot{\boldsymbol{\omega}}=\nabla^{A} \mathbf{v}=\frac{1}{2}(\nabla \otimes \mathbf{v}-\mathbf{v} \otimes \nabla)
$$

Applying the SPH method the equations (19) and (21) become the equations (22) and (23) [13].

$$
\begin{aligned}
& \langle\dot{\boldsymbol{\varepsilon}}\rangle=\frac{1}{2} \sum_{j=1}^{N} \frac{m_{j}}{\rho_{j}}\left[\nabla_{i} W_{i j} \otimes\left(\mathbf{v}_{j}-\mathbf{v}_{i}\right)+\left(\mathbf{v}_{j}-\mathbf{v}_{i}\right) \otimes \nabla_{i} W_{i j}\right] \\
& \langle\dot{\boldsymbol{\omega}}\rangle=\frac{1}{2} \sum_{j=1}^{N} \frac{m_{j}}{\rho_{j}}\left[\nabla_{i} W_{i j} \otimes\left(\mathbf{v}_{j}-\mathbf{v}_{i}\right)-\left(\mathbf{v}_{j}-\mathbf{v}_{i}\right) \otimes \nabla_{i} W_{i j}\right]
\end{aligned}
$$

This set of equations can now be solved and will describe a perfectly elastic materials behavior. However, it is known that studied materials are not only perfectly elastic but instead present also a plastic behavior, for this a critical stress always exists and the employment of 
such was used for the material in permanent deformation. This plastic behavior can be introduced in the equations using the von Mises yielding criterion [20], [21], and the deviatoric stress is limited by a scalar function $\tilde{f}$ defined by (24).

$$
\tilde{\boldsymbol{\sigma}}=\tilde{f} \tilde{\boldsymbol{\sigma}}
$$

Plasticity in the von Mises yielding criterion is tread using the Jhonson and Cook viscoplastic model [12] showing in (25). It depends on the plastic strain, the plastic strain rate and temperature variations.

$$
\begin{aligned}
& Y_{J C}=\left[A+B\left(\varepsilon_{e q}\right)^{n}\right]\left[1+C \ln \left(\frac{\dot{\varepsilon}_{e q}}{\dot{\varepsilon}_{0}}\right)\right]\left[1-\left(\frac{T-T_{0}}{T_{m}-T_{0}}\right)^{m}\right] \\
& \varepsilon_{e q}=\frac{2}{3} \sqrt{\frac{3}{2} \tilde{\boldsymbol{\varepsilon}}: \tilde{\boldsymbol{\varepsilon}}} \\
& \tilde{\boldsymbol{\varepsilon}}=\boldsymbol{\varepsilon}-\operatorname{tr}(\boldsymbol{\varepsilon}) \mathbf{1} \\
& \dot{\varepsilon}_{e q}=\frac{2}{3} \sqrt{\frac{3}{2} \dot{\tilde{\boldsymbol{\varepsilon}}}: \dot{\tilde{\boldsymbol{\varepsilon}}}} \\
& \dot{\tilde{\boldsymbol{\varepsilon}}}=\dot{\boldsymbol{\varepsilon}}-\operatorname{tr}(\dot{\boldsymbol{\varepsilon}})
\end{aligned}
$$

Where the equations (25) to (27), $A$ is the initial yield stress ( $M P a), B$ is the coefficient of strength $(M P a), n$ is the work hardening exponent, $C$ is the coefficient of strain rate sensitivity, $p$ is the thermal softening coefficient, $\dot{\varepsilon}_{0}$ is the strain rate reference value (defined as $\left.\dot{\varepsilon}_{0}=1\right), T$ is the material temperature $(K), T_{0}$ is the room temperature, $T_{m}$ is the material melting temperature, $\tilde{\boldsymbol{\varepsilon}}$ is the deviatoric strain, $\varepsilon_{e q}$ is the equivalent plastic strain and $\dot{\varepsilon}_{e q}$ is the equivalent plastic strain rate, the two last ones are defined in the equations (26) and (27) respectively. In this work the test problems have a high strain behavior, by such circumstances it is entirely reasonable that when yielding criterion is reached, the assumption that the elastic strain is zero and the plastic strain becomes the total strain can be made; according to this the function $\tilde{f}$ (28) can now be computed following the widely used form [2], [6], [22], where $\sigma_{e q}$ is equivalent stress, which is given by (29).

$$
\begin{aligned}
& f=\min \left[\frac{\left(Y_{J C}\right)^{2}}{\left(\sigma_{e q}\right)^{2}}, 1\right] \\
& \sigma_{e q}=\sqrt{\frac{3}{2} \tilde{\boldsymbol{\sigma}}: \tilde{\boldsymbol{\sigma}}}
\end{aligned}
$$

\subsection{Model's definition}

For the time stepping we used the Predictor-Corrector algorithm described by Monaghan [23] because presents more stability for the variables time update. 
The calculations for boundary conditions in this work were made with boundaries defined by lines that exert repulsive force [4], [23], [24], this approach improves the physical behavior because produces a force normal to the boundary over the neighboring particles [25], [26].

Further, we used other improvements like the XSPH [23] for moving the particles, Moving Least Squares (MLS) [27], [28] for density re-initialization and Artificial stress [8] for treatment of instability tensile.

\section{NUMERICAL TESTS}

Examples for simulating high strain with material strength were carried out using SPH. The SPH results were compared with the result of the other SPH works and experimental data. In this section are presented three test, many employed in the literature [5], [29], [30]. The study examples are high shear in a plate, the impact of a plate against a rigid surface and the penetration of a cylinder through a plate.

\subsection{High Shear plate}

This test was develop in [5], [29] at first. In this example, an Armco iron plate in two dimensions, the upper half of the plate has an initial velocity of $254 \mathrm{~m} / \mathrm{s}$, while the lower bottom starts at rest as seen in Figure 1. The material properties and the parameters for MieGruneisen equation of the Armco iron are presented in Table 1, and in Table 2 are shown the parameters of the Johnson and Cook model and the artificial viscosity coefficient. In this case 1122 particles were initially distributed to represent the plate.

In Figure 2 and Figure 3 is showed a comparison between temperature and equivalent strain at $80 \mu \mathrm{s}$ of simulation. The Figure 2 and Figure 3 shows that the maximum strain and temperature are found in the shear plane between the lower and the upper half, from these behaviors it can be intuited that the model is appropriate. In Figure 4 are compared our SPH simulation with that made by Gordon R. Johnson [5], [29], it can be seen that our simulation ( Figure 4(a)) shows a more realistic behavior in the interaction between particles compared with Figure 4 (b) in which the material exhibits instability in the separation plane, this improvement is due to the implementation of the artificial viscosity [29], XSPH [23] and artificial stress [22]. While the comparison between our SPH model and the standard finite element method (FEM) (Figure 4 (c)), shows that SPH presents a more rigid behavior in deformation and distributes the strain uniformly in all the particles.

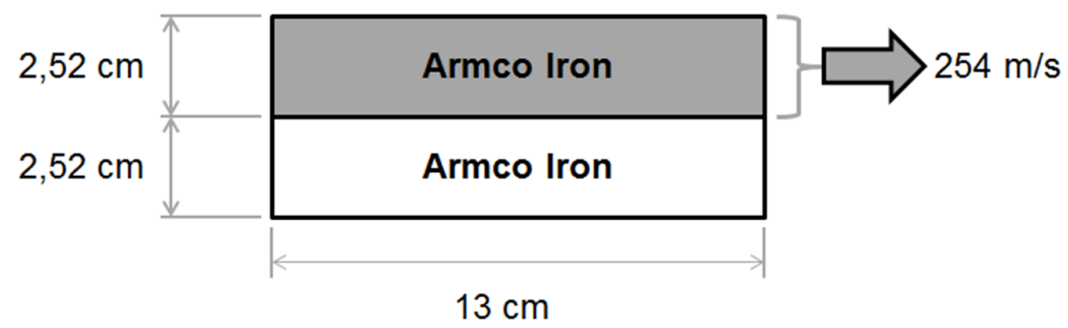

Figure 1. Test 1 high Shear plate, initial conditions.

Table 1.The material properties and the parameters for Mie-Gruneisen equation of the Armco iron [13].

\begin{tabular}{|c|c|c|c|c|c|}
\hline$\mu[\mathrm{GPa}]$ & $C_{p}[\mathrm{~J} / \mathrm{kgK}]$ & $k[\mathrm{~W} / \mathrm{mK}]$ & $\rho\left[\mathrm{kg} / \mathrm{m}^{3}\right]$ & $\Gamma$ & $S_{s}$ \\
\hline 80 & 452 & 80,2 & 7890 & 1,81 & 1,8 \\
\hline
\end{tabular}


Table 2.The parameter in Armco iron for the Johnson and Cook model and coefficient in artificial viscosity [12].

\begin{tabular}{|c|c|c|c|c|c|c|c|c|}
\hline$A[M p a]$ & $B[M P a]$ & $C$ & $n$ & $m$ & $T_{0}[K]$ & $T_{m}[K]$ & $\alpha$ & $\beta$ \\
\hline 175 & 380 & 0,060 & 0,32 & 0,55 & 293,15 & 1811 & 0,2 & 4 \\
\hline
\end{tabular}

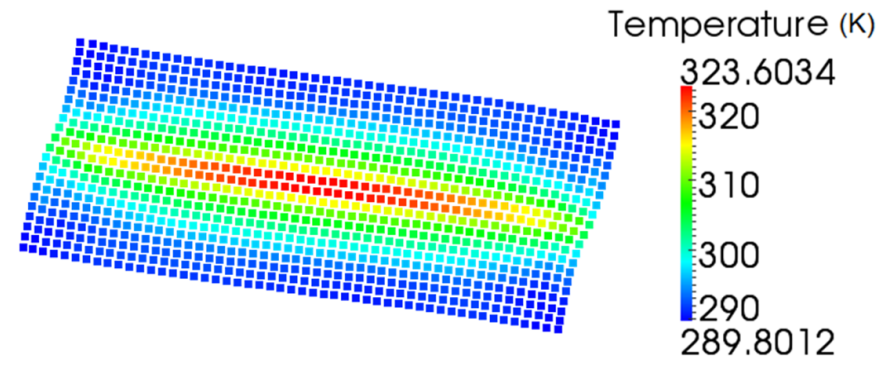

Figure 2. High Shear plate, Temperature distribution at $80 \mu s$.

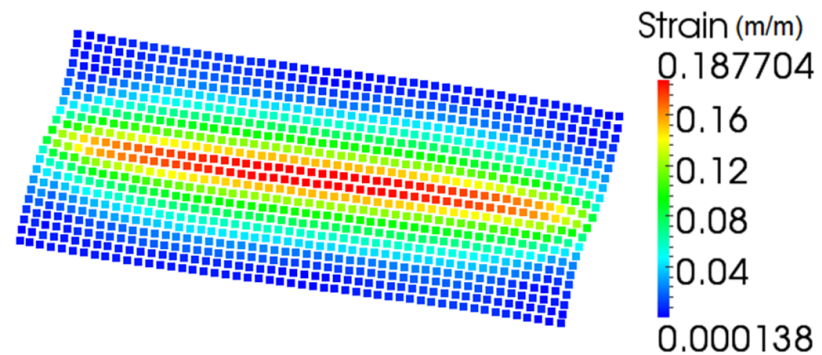

Figure 3. High Shear plate, strain distribution at $80 \mu \mathrm{s}$.

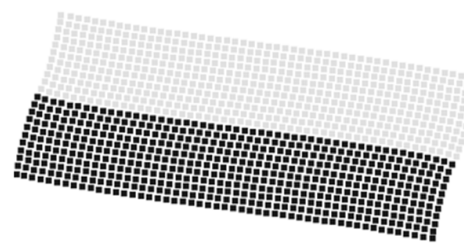

(a)

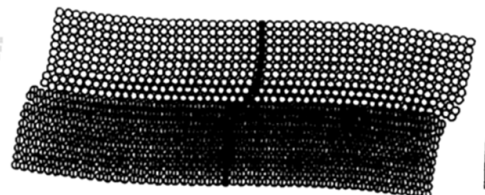

(b)

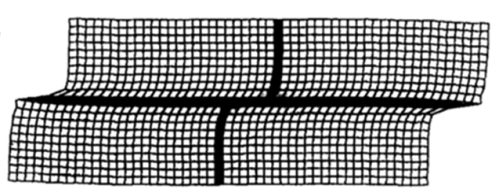

(c)

SPH result by Patiño, Reyes et al. SPH result by Gordon R. Johnson. FEM result by Gordon R. Johnson

Figure 4. Compared the SPH simulation made in this work with the made by Gordon R. Johnson (at $80 \mu s$ ).

\subsection{Impact of a plate against a rigid surface}

This problem has been widely used in the literature [6], [29], [30]. An Armco iron cylinder represented with a plate in two dimensions is traveling at $200 \mathrm{~m} / \mathrm{s}$ and in impacts on a rigid static surface. The plate was $2,546 \mathrm{~cm}$ longitude, and $0,76 \mathrm{~cm}$ wide (Figure 5). The material properties and parameters of models for the Armco iron are presented in the Table 1 and Table 2. The separation between real particles was $300 \mu \mathrm{m}$.

The Figure 6 shows the final deformed shape of the cylinder and its dimension and the

Table 3 is the comparison between the SPH simulation made in this work and the made by Gordon R. Johnson [29], Libersky [6] and Liu [30] . From Figure 6 and 
Table 3 it can be seen a good behaviour of our model compared to previous SPH models presented in the literature.

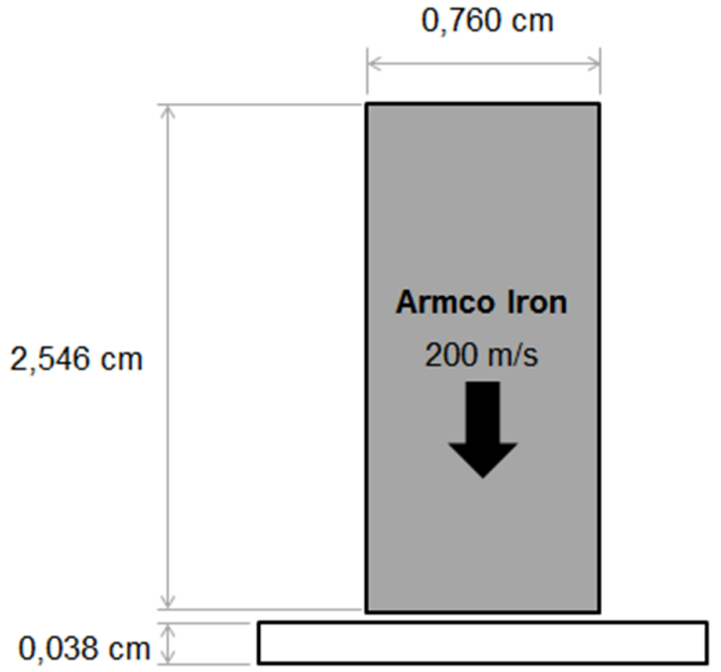

Figure 5. The impact of a plate against a rigid surface, initial condition.

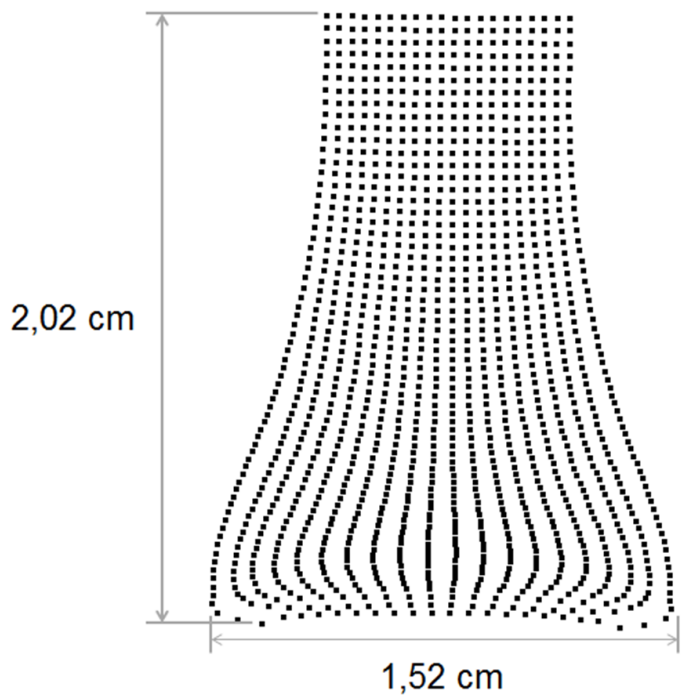

Figure 6 . The impact of a plate against a rigid surface, final condition.

Table 3. Comparing our SPH simulation of "The impact of a plate against a rigid surface" with that made by Libersky [6], Liu [30] and Gordon R. Johnson [29].

\begin{tabular}{|c|c|c|}
\hline Impact cylinder SPH Models & Final bar height (cm) & Final bar width (cm) \\
\hline Result by Patiño, Reyes et al. & 2,02 & 1,52 \\
\hline Result by Libersky [6] & 2,18 & 1,52 \\
\hline Result by Liu [30] & 2,09 & 1,93 \\
\hline Result by Johnson [29] & 2,11 & 1,70 \\
\hline
\end{tabular}

\subsection{Penetration of a cylinder through a plate}

In this case, the model was applied to simulate the penetrating process of an infinitely long aluminum cylinder through an infinitely long aluminum plate, this problem has been widely studied [3], [5], [29]. The simulation has two dimensions, taken as the transversal sec- 
tion of both cylinder and plate. Figure 7 presents the initial conditions, while the material properties and the parameters for Mie-Gruneisen equation for the Aluminum are presented in Table 4. Table 5 shows the parameter for the Johnson and Cook model and the artificial viscosity coefficient. In this case 12830 particles were initially distributed. The impact speed of the cylinder was $6180 \mathrm{~m} / \mathrm{s}$ and the simulations were run up to $20 \mu \mathrm{s}$.

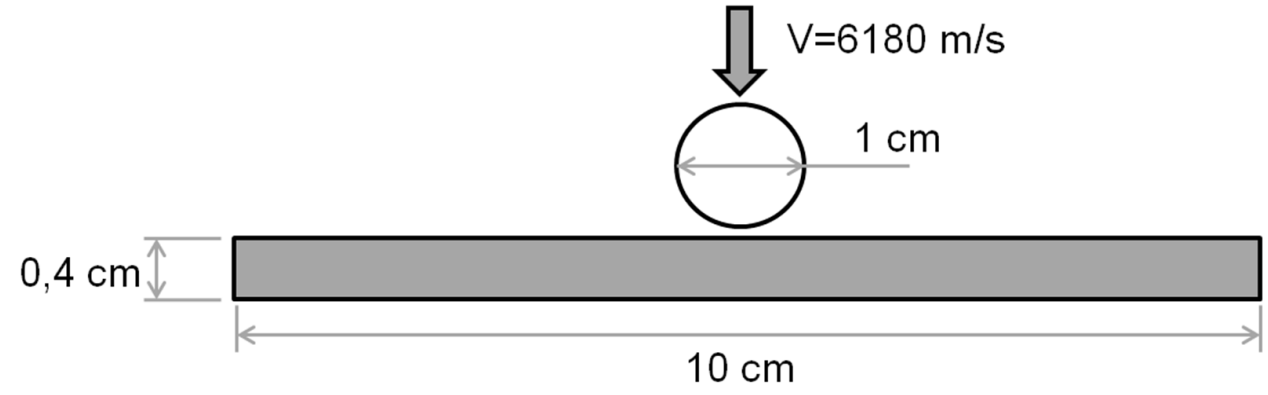

Figure 7 . The penetration of a cylinder through a plate, initial conditions.

Table 4 . The material properties and the parameters for Mie-Gruneisen equation of the Aluminum [9], [18].

\begin{tabular}{|c|c|c|c|c|c|}
\hline$\mu[\mathrm{GPa}]$ & $C_{p}[\mathrm{~J} / \mathrm{kgK}]$ & $k[\mathrm{~W} / \mathrm{mK}]$ & $\rho\left[\mathrm{kg} / \mathrm{m}^{3}\right]$ & $\Gamma$ & $S_{s}$ \\
\hline 27,1 & 875 & 237 & 2710 & 1,7 & 1,5 \\
\hline
\end{tabular}

Table 5.The parameter in Aluminum for the Johnson and Cook model and coefficient in artificial viscosity [9].

\begin{tabular}{|c|c|c|c|c|c|c|c|c|}
\hline$A[M p a]$ & $B[M P a]$ & $C$ & $n$ & $m$ & $T_{0}[K]$ & $T_{m}[K]$ & $\alpha$ & $\beta$ \\
\hline 300 & 426 & 0,015 & 0,34 & 1 & 273,15 & 775 & 2,5 & 2,5 \\
\hline
\end{tabular}

The strain in the cylinder and the plate at $20 \mu s$ can be seen in Figure 8 and Table 6 , the comparison between the results of the simulation with the results obtained by Liu [30] and S. Hiermaier [9] shows an adequate behavior.

The Figure 9 and Figure 10 present the initial impact behavior, showing the equivalent strain and equivalent strain rate, these show a high plastic strains as it should be assumed in the impact test. It is important to say that the deformation behaviour and the strain rate are distributed over the contact and penetration between the cylinder and the plate, which is physically correct, however the model presents some unnatural separations in the cylinder, probably because of the bad interaction between particles at the penetration starting instant. The fracture criterion was only the natural separation between particles [3].

Table 6. Compared simulation behavior in this paper with the made by Liu [30] and S. Hiermaier [9] at $20 \mu s$.

\begin{tabular}{|c|c|c|c|c|}
\hline $\begin{array}{c}\text { Cylinder and } \\
\text { plate }\end{array}$ & $\begin{array}{c}\text { Patiño, Reyes } \\
\text { et. al }\end{array}$ & $\begin{array}{c}\text { G.R. Liu } \\
{[30]}\end{array}$ & $\begin{array}{c}\text { S. HIER- } \\
\text { MAIER [9] }\end{array}$ & $\begin{array}{c}\text { Experimental } \\
{[9]}\end{array}$ \\
\hline $\mathrm{Dg}(\mathrm{cm})$ & 3,2 & 3,35 & 3,5 & $2,75-3,45$ \\
\hline $\mathrm{L} / \mathrm{w}$ & 1,388 & 1,33 & 1,11 & 1,39 \\
\hline
\end{tabular}




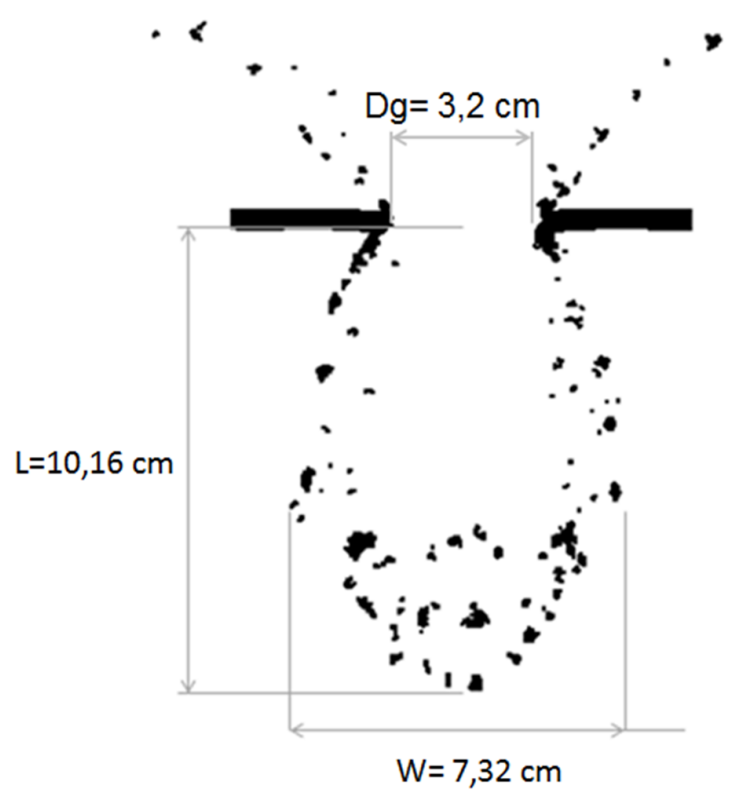

Figure 8 . The strain in the cylinder and plane by $20 \mu s$.

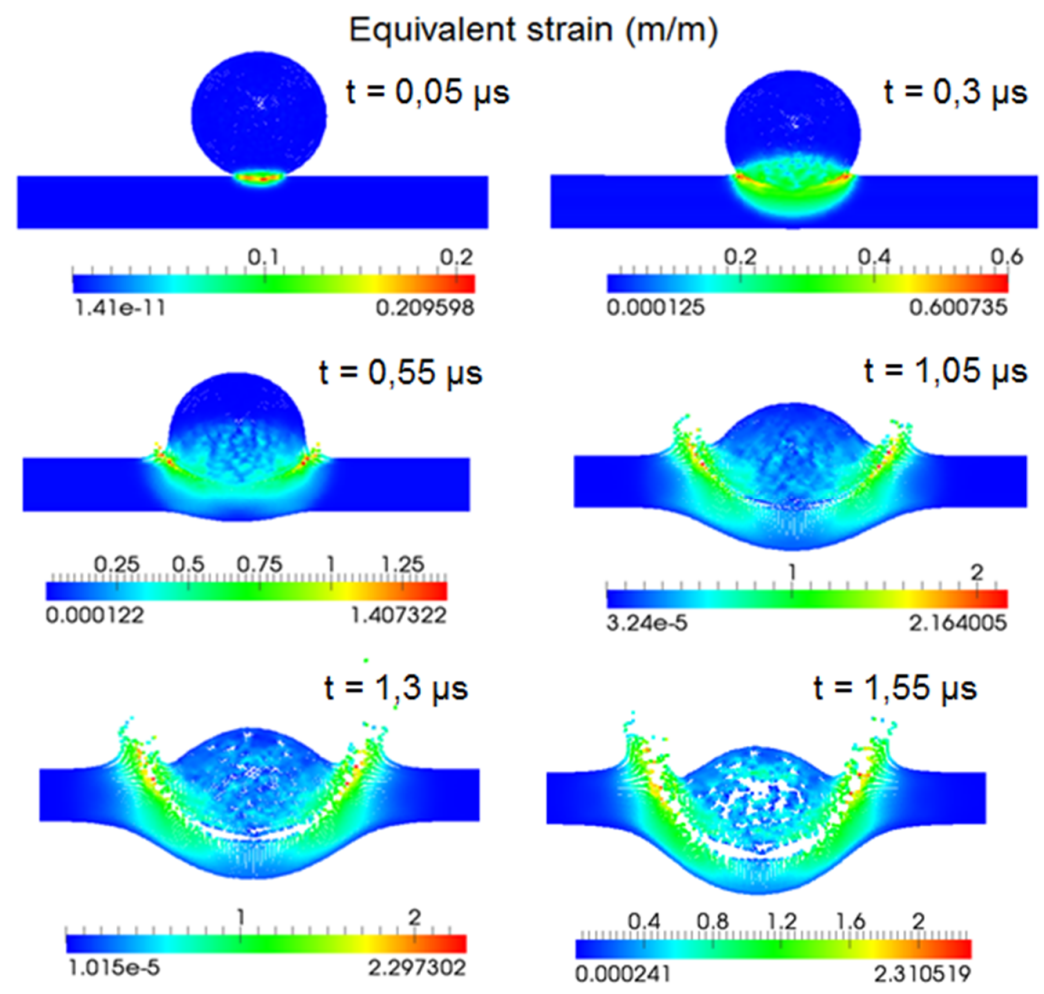

Figure 9. Equivalent strain by $1,55 \mu \mathrm{s}$. 


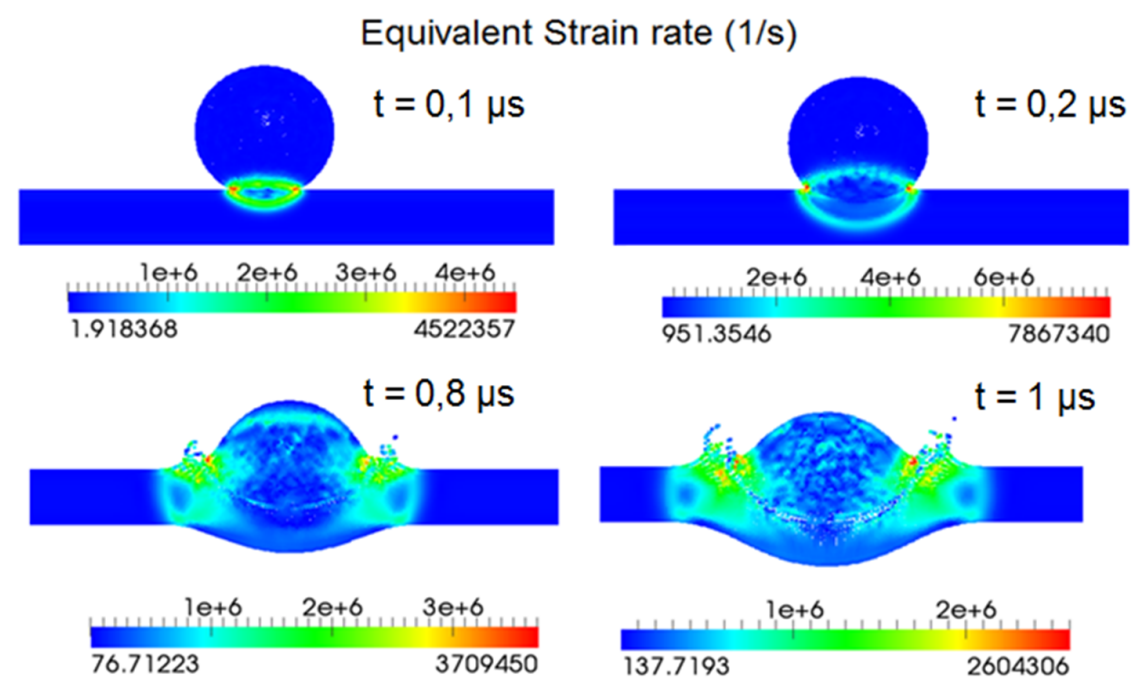

Figure 10. Equivalent strain rate by $1 \mu \mathrm{s}$.

\section{CONCLUSIONS}

We presented three two-dimensional models of materials strain by the method of Smoothed Particle Hydrodynamics (SPH) for high strains, considering elastic deformations by Hooke's law and plastic strains through the yielding criterion of von Mises and the Johnson-Cook model.

The test of High Shear plate shows an adequate behaviour in the interaction between particles in our SPH model compared to other models and experimental data. The impact of a rigid plate against a surface presents realistic behavior between particles and rigid boundaries as compared with other simulations. Penetration of a cylinder through a plate has a good performance relative to other models and experimental values, but we still have some irregular separations at the penetration time.

The functionality of SPH for high strain processes modeling was proved and the examples developed showed an appropriate behavior with respect to other models and experimental data.

In future implementations an improvement in the fracture criterion to represent the separation between particles in a better way is necessary

\section{REFERENCE}

[1] J. J. Monaghan, "Smoothed Particle Hydrodynamics," Annual Review of Astronomy and Astrophysics, vol. 30, no. 1, pp. 543-574, Sep. 1992.

[2] W. Benz and E. Asphaug, "Simulations of brittle solids using smooth particle hydrodynamics," Computer Physics Communications, vol. 87, no. 1-2, pp. 253-265, May 1995.

[3] P. Randles and L. D. Libersky, "Smoothed particle hydrodynamics: some recent improvements and applications," Computer methods in applied mechanics and, vol. 7825, no. 96, 1996.

[4] J. J. Monaghan, "Smoothed Particle Hydrodynamics and Its Diverse Applications," Annual Review of Fluid Mechanics, 2012. 
[5] G. R. Johnson, R. a. Stryk, and S. R. Beissel, "SPH for high velocity impact computations," Computer Methods in Applied Mechanics and Engineering, vol. 139, no. 1-4, pp. 347-373, Dec. 1996.

[6] L. D. Libersky and A. G. Petschek, Advances in the Free-Lagrange Method Including Contributions on Adaptive Gridding and the Smooth Particle Hydrodynamics Method, vol. 395. Berlin, Heidelberg: Springer Berlin Heidelberg, 1991, pp. 248-257.

[7] P. W. Cleary and R. Das, "The Potential for SPH Modelling of Solid Deformation and Fracture," Media, pp. 287-296.

[8] J. P. Gray, J. J. Monaghan, and R. P. Swift, "SPH elastic dynamics," Computer Methods in Applied Mechanics and Engineering, vol. 190, no. 49-50, pp. 6641-6662, Oct. 2001.

[9] S. Hiermaier, D. Konke, A. J. J. Stilp, K. Thoma, and D. Könke, "Computational simulation of the hypervelocity impact of al-spheres on thin plates of different materials," International Journal of Impact Engineering, vol. 20, no. 1-5, pp. 363-374, Jan. 1997.

[10] P. Cleary, M. Prakash, and J. Ha, "Novel applications of smoothed particle hydrodynamics (SPH) in metal forming," Journal of Materials Processing Technology, vol. 177, no. 1-3, pp. 41-48, Jul. 2006.

[11] J. Limido, C. Espinosa, M. Salau, and J. L. Lacome, "SPH method applied to high speed cutting modelling," International Journal of Mechanical Sciences, vol. 49, pp. 898-908, 2007.

[12] G. R. Johnson and W. H. Cook, "A constitutive model and data for metals subjected to large strains, high strain rates and high temperatures," Proceedings of the 7th International Symposium on Ballistics, vol. 547, no. 11. the hague, Netherlands, pp. 541-547, 1983.

[13] G. R. Liu and M. B. Liu, Smoothed particle hydrodynamics: a meshfree particle method. Singapore: World Scientific Publishing Co., 2003.

[14] M. B. Liu and G. R. Liu, "Smoothed Particle Hydrodynamics (SPH): an Overview and Recent Developments," Archives of Computational Methods in Engineering, vol. 17, no. 1, pp. 25-76, 2010.

[15] J. J. Monaghan, "Smoothed particle hydrodynamics," Reports on Progress in Physics, vol. 68, no. 8, pp. 1703-1759, Aug. 2005.

[16] R. Capuzzo-Dolcetta and R. Di Lisio, "Criterion for the choice of the interpolation kernel in smoothed particle hydrodynamics," Applied Numerical Mathematics, vol. 34, no. 4, pp. 363-371, Aug. 2000.

[17] R. Gingold and J. Monaghan, "Kernel estimates as a basis for general particle methods in hydrodynamics," Journal of Computational Physics, vol. 46, no. 3, pp. 429-453, Jun. 1982. 
[18] P. Randles, T. Carney, and L. Libersky, "Calculation of oblique impact and fracture of tungsten cubes using smoothed particle hydrodynamics," journal of impact, vol. 17, pp. 661-672, 1995.

[19] P. W. Cleary and J. J. Monaghan, "Conduction Modelling Using Smoothed Particle Hydrodynamics,” vol. 264, pp. 227-264, 1999.

[20] F. Dunne and P. N, Introduction to computational plasticity. Oxford University Press, 2005.

[21] E. A. de S. Neto, D. Peric, and D. R. J. Owen, Computational methods for plasticity: theory and applications. Swansea: WILEY A John and Sons,Ltd, 2008.

[22] J. P. Gray and J. J. Monaghan, "Numerical modelling of stress fields and fracture around magma chambers," Journal of Volcanology and Geothermal Research, vol. 135, pp. 259-283, 2004.

[23] J. J. Monaghan, "On the problem of penetration in particle methods," Journal of Computational Physics, vol. 82, no. 1, pp. 1-15, May 1989.

[24] J. J. Monaghan and A. Kos, "Solitary Waves on a Cretan Beach," Journal of Waterway Port Coastal and Ocean Engineering, vol. 125, no. June, pp. 145-154, 1999.

[25] Y. Amini, H. Emdad, and M. Farid, "A new model to solve fluid-hypo-elastic solid interaction using the smoothed particle hydrodynamics (SPH) method," European Journal of Mechanics - B/Fluids, vol. 30, no. 2, pp. 184-194, Mar. 2011.

[26] S. Seo, O. Min, and J. Lee, "Application of an improved contact algorithm for penetration analysis in SPH," International Journal of Impact Engineering, vol. 35, no. 6, pp. 578-588, Jun. 2008.

[27] G. A. Dilts, "Moving-least-squares-particle hydrodynamics I. Consistency and stability," International Journal for Numerical Methods in Engineering, vol. 44, no. 8, pp. 1115-1155, Mar. 1999.

[28] G. A. Dilts, "Moving least-squares particle hydrodynamics II: conservation and boundaries," International Journal for Numerical Methods in Engineering, vol. 48, no. 10, pp. 1503-1524, Aug. 2000.

[29] G. R. Johnson, "Artificial viscosity effects for SPH impact computations," International Journal of Impact Engineering, vol. 18, no. 5, pp. 477-488, Jul. 1996.

[30] M. B. Liu, G. R. Liu, and K. Y. Lam, "Adaptive smoothed particle hydrodynamics for high strain hydrodynamics with material strength," Shock Waves, vol. 15, no. 1, pp. 2129, Jan. 2006. 